\title{
Effects of Unilateral Nephrectomy on Renal Function in Male Spontaneously Diabetic Torii Fatty Rats: A Novel Obese Type 2 Diabetic Model
}

\author{
Yoshiaki Katsuda, ${ }^{1}$ Yusuke Kemmochi, ${ }^{2}$ Mimi Maki, ${ }^{1}$ Ryuhei Sano, ${ }^{1}$ Yasufumi Toriniwa, ${ }^{1}$ \\ Yukihito Ishii, ${ }^{1}$ Katsuhiro Miyajima, ${ }^{2}$ Kochi Kakimoto, ${ }^{2}$ and Takeshi Ohta ${ }^{1}$ \\ ${ }^{1}$ Japan Tobacco Inc., Central Pharmaceutical Research Institute, Biological/Pharmacological Research Laboratories, 1-1 Murasaki-cho, \\ Takatsuki, Osaka 569-1125, Japan \\ ${ }^{2}$ Japan Tobacco Inc., Central Pharmaceutical Research Institute, Toxicology Research Laboratories, 23 Naganuki, \\ Hadano, Kanagawa 257-0024, Japan
}

Correspondence should be addressed to Takeshi Ohta; takeshi.ota@jt.com

Received 23 June 2014; Accepted 7 July 2014; Published 10 August 2014

Academic Editor: Masami Shinohara

Copyright (C) 2014 Yoshiaki Katsuda et al. This is an open access article distributed under the Creative Commons Attribution License, which permits unrestricted use, distribution, and reproduction in any medium, provided the original work is properly cited.

\begin{abstract}
The Spontaneously Diabetic Torii (SDT) fatty rat is a new model for obese type 2 diabetes. The aim of the present study was to investigate the effect of $1 / 2$ nephrectomy $(\mathrm{Nx})$ on renal function and morphology and on blood pressure in SDT fatty rats. Male SDT fatty rats underwent $1 / 2 \mathrm{Nx}$ or a sham operation (Sham). Subsequently, animals were studied with respect to renal function and histological alterations. Induction of $1 / 2 \mathrm{Nx}$ in SDT fatty rats led to functional and morphological damage to the remnant kidney and to hypertension, which are considered main characteristics of chronic kidney disease, at a younger age compared with the sham group. In conclusion, the SDT fatty rat is useful in investigations to elucidate the pathogenesis of human diabetic nephropathy and in new drug discovery.
\end{abstract}

\section{Introduction}

Chronic kidney disease (CKD) is a worldwide public health problem associated with significant morbidity and mortality [1]. Several risk factors contribute to the development and progression of $\mathrm{CKD}$, including hypertension, diabetes, and dyslipidemia [2-4]. In particular the increase in number of patients with obesity-associated type 2 diabetes has resulted in a rapid increase in patients who have end stage renal disease (ESRD) and require dialytic life support $[5,6]$. Despite efforts to develop means to prevent or arrest the progression of the disease, long-term prognosis of patients with established nephropathy remains poor [7]. Diabetic nephropathy has been recognized as a primary disease of CKD and the investigation of diabetic nephropathy is essential for the understanding of the pathogenesis of CKD.
Diabetic animal models have a critical role in the elucidation of mechanisms of diabetic complications and the development of novel drugs as treatments. Consequently, the understanding of the pathophysiology of renal lesions in diabetic models is beneficial in the design and development of therapies.

The Spontaneously Diabetic Torii (SDT) fatty rat, established by introducing the $f a$ allele in Zucker fatty rats into the SDT rat genome, is a model for obese type 2 diabetes showing overt obesity, hyperglycemia, and hyperlipidemia from a younger age and resulting in early onset of diabetic complications [8-12]. Furthermore, SDT fatty rats showed elevated blood pressure, in addition to the aforementioned metabolic abnormalities $[13,14]$. Therefore, the SDT fatty rat is considered to be a useful model for the analysis of diabetes and related complications such as diabetic nephropathy. 
In this study, we investigated the effects of unilateral nephrectomy on renal function and morphology in SDT fatty rats.

\section{Materials and Methods}

2.1. Animals. This experiment was conducted in compliance with the Guidelines for Animal Experimentation established for our biological/pharmacological laboratories. Male SDT fatty rats from Japan Tobacco colonies were used in this study. Animals were divided into 2 groups: those undergoing 1/2 nephrectomy $(1 / 2 \mathrm{Nx})$ or sham operation (sham). Animals at 8 weeks of age underwent $1 / 2 \mathrm{Nx}$ or sham surgery under anesthesia. A small lumbar incision was made, and the left kidney was removed. In sham-operated animals, the left kidney was exposed and gently manipulated but left intact. Animals were housed in suspended bracket cages and given a standard laboratory diet (CRF-1; Oriental Yeast Co., Ltd., Tokyo, Japan) in a room with controlled temperature, humidity, and lighting.

2.2. Biological Parameters. Body weight, biochemical parameters, and renal parameters were assessed from 10 to 18 weeks of age, every 2 weeks. Blood samples were collected from the tail vein of nonfasted rats. Serum glucose, triglyceride (TG), and total cholesterol (TC) were measured as a biochemical parameter using commercial kits (F. Hoffmann-La Roche Ltd., Basel, Switzerland) and an automatic analyzer (Hitachi, Ltd., Tokyo, Japan).

Urine volume, urinary albumin, blood urea nitrogen (BUN), and creatinine clearance (Ccr) were evaluated as renal parameters. Urine samples were collected by placing the animals in metabolic cages with water for $6 \mathrm{~h}$. Urinary albumin level was measured with a rat-albumin enzyme immunoassay (EIA) kit (Panapharm Laboratories Co., Ltd., Kumamoto, Japan). Urinary creatinine, serum creatinine, and BUN were measured using commercial kits (Roche Diagnostics, Basel, Switzerland) and an automatic analyzer (Hitachi, Ltd., Tokyo, Japan). Ccr was calculated by dividing the $6 \mathrm{~h}$ urinary excretion of creatinine by serum creatinine level and body weight.

Systolic blood pressure (SBP) and heart rate in a conscious nonfasted state were measured at 12 and 16 weeks of age by the indirect tail cuff method using a Softron BP98A indirect blood pressure meter (Softron Co. Ltd., Tokyo, Japan). Blood pressure and heart rate were measured between 13:00 and 16:00 hours. Five measurements were taken for each rat and subsequently averaged.

2.3. Histopathological Examination. Necropsy was performed at 18 weeks of age and kidneys were collected from all animals. Kidneys were weighed and fixed with $4 \%$ paraformaldehyde. After resection, the tissues were paraffinembedded using standard techniques and thin-sectioned (3-5 $\mu \mathrm{m})$. Sections were stained with hematoxylin and eosin (HE) and periodic acid Schiff (PAS). Eight mice in the 1/2 $\mathrm{Nx}$ group and five mice in the sham group were examined histopathologically in a blinded manner.
2.4. Statistical Analysis. Results of biological parameters were expressed as means \pm standard deviation (SD). Statistical analyses of differences between mean values in the sham group and the 1/2 Nx group were performed using the $F$-test, followed by the Student's $t$-test or Aspin-Welch's $t$-test. Differences were defined as significant when $P<0.05$.

\section{Results}

3.1. Body Weight and Biochemical Parameters. Body weights in the $1 / 2 \mathrm{Nx}$ group were comparable to those in the sham group from 10 to 18 weeks of age ( $1 / 2 \mathrm{Nx}$ group; $479.7 \pm 116.2 \mathrm{~g}$ at 18 weeks of age, sham group; $521.2 \pm 40.3 \mathrm{~g}$ at 18 weeks of age). The $1 / 2 \mathrm{Nx}$ group and sham group had similar levels of plasma glucose from 10 to 18 weeks of age (1/2 Nx group, $751.0 \pm 63 \mathrm{mg} / \mathrm{dL}$ at 18 weeks of age; sham group, $799.9 \pm$ $76.9 \mathrm{mg} / \mathrm{dL}$ at 18 weeks of age). Serum TG levels in the $1 / 2 \mathrm{Nx}$ group at 18 weeks of age were significantly higher than those in the sham group (1/2 Nx group, $501.9 \pm 211.4 \mathrm{mg} / \mathrm{dL}$ at 18 weeks of age; sham group, $309.6 \pm 67.4 \mathrm{mg} / \mathrm{dL}$ at 18 weeks of age). Serum TC levels tended to increase in the $1 / 2 \mathrm{Nx}$ group (1/2 Nx group, $154.4 \pm 51.7 \mathrm{mg} / \mathrm{dL}$ at 18 weeks of age; sham group, $120.8 \pm 18.7 \mathrm{mg} / \mathrm{dL}$ at 18 weeks of age).

3.2. Renal Parameters. Urinary albumin in the $1 / 2 \mathrm{Nx}$ group increased from 14 weeks of age compared with those in the sham group (Figure 1(a)). Serum BUN levels in the $1 / 2 \mathrm{Nx}$ group were significantly higher than those in the sham group during the experimental period (Figure 1(b)). Kidney weights in the $1 / 2 \mathrm{Nx}$ group were higher compared with those in the sham group (Figure $1(\mathrm{c})$ ). Ccr tended to decrease in the $1 / 2 \mathrm{Nx}$ group (1/2 Nx group, $0.23 \pm 0.12 \mathrm{~mL} / \mathrm{h} * \mathrm{~g}$ at 18 weeks of age; sham group, $0.31 \pm 0.06 \mathrm{~mL} / \mathrm{h} * \mathrm{~g}$ at 18 weeks of age). Urine volumes in the $1 / 2 \mathrm{Nx}$ group were comparable to those in the sham group from 10 to 18 weeks of age (1/2 Nx group, $13.17 \pm 6.58 \mathrm{~mL}$ at 18 weeks of age, $0.23 \pm 0.12 \mathrm{~mL} / \mathrm{h} * \mathrm{~g}$; sham group, $15.90 \pm 6.11 \mathrm{~mL}$ at 18 weeks of age).

3.3. Blood Pressure. SBP levels at 12 and 16 weeks of age in the $1 / 2$ Nx group were significantly elevated compared with those in the sham group (Figure 2(a)). For heart rate, there were no differences among groups (Figure 2(b)).

3.4. Histopathological Examinations of the Kidney. The results of histopathological examinations of the kidney at 18 weeks of age are shown in Table 1 and Figure 3. The following findings in the glomerulus, tubule, and interstitium were observed in both the sham group and 1/2 Nx group. Glomerulosclerosis was characterized by an increase in size of the glomerulus and diffuse thickening of the glomerulocapillary wall and the mesangial expansion, showing partly segmental fibrosis in severe cases. Tubular lesions included tubular regeneration, dilatation, and hyaline casts, and interstitial lesions included fibrosis and inflammatory cell infiltration. The histological features of the kidney were not different between the $1 / 2$ Nx group and the sham group; however, a more progressive pathological degree was observed in the $1 / 2 \mathrm{Nx}$ group. 


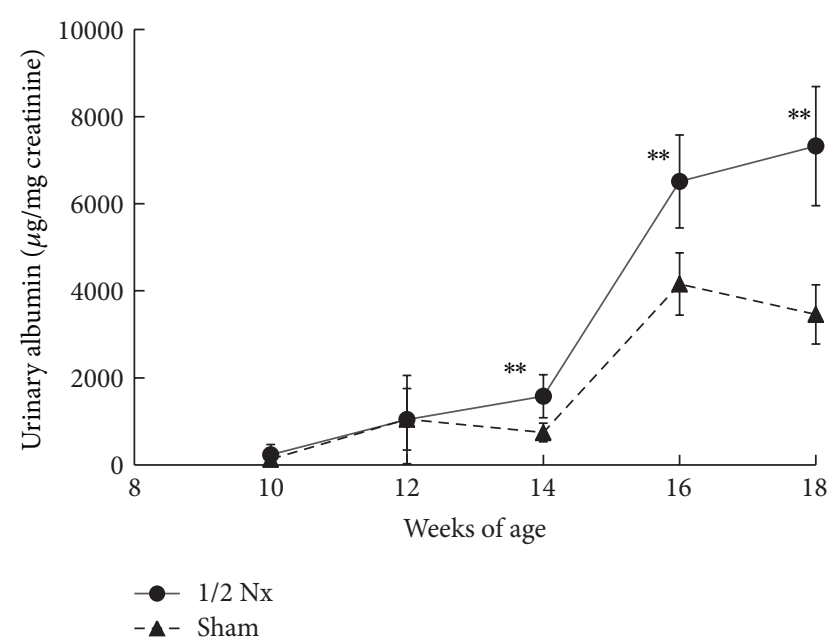

(a)

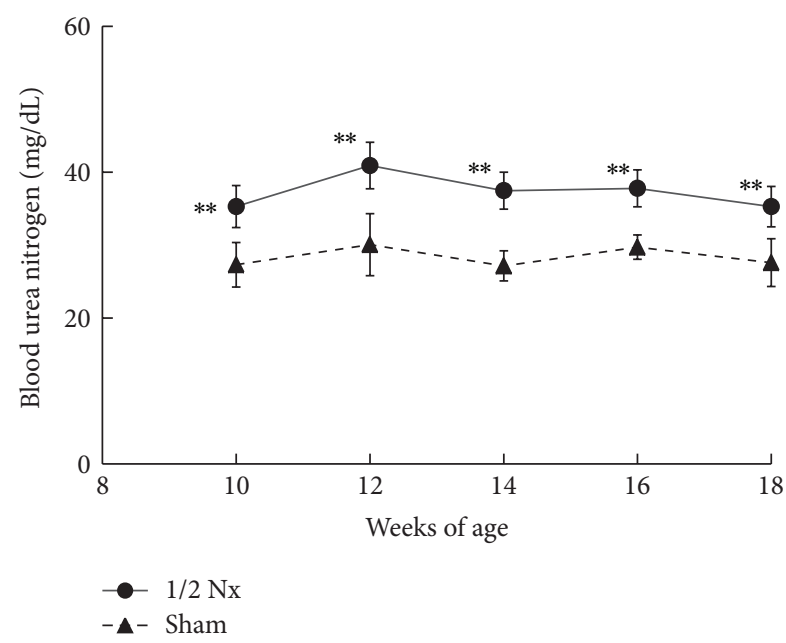

(b)

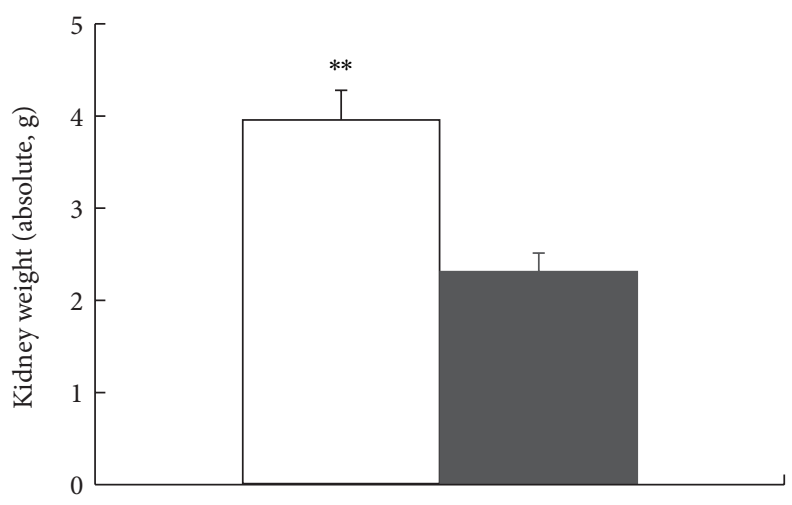

Figure 1: Changes in renal parameters ((a) urinary albumin, (b) blood urea nitrogen, and (c) kidney weight) in the 1/2 Nx group and sham group. Data shown as means $\pm \mathrm{SD}((\mathrm{a})$ and $(\mathrm{b}) n=8-10$ and $(\mathrm{c}) n=8-9) .{ }^{* *} P<0.01$; significantly different from the sham group.

\section{Discussion}

Glomerular lesions occurring due to renal mass reduction have been demonstrated $[15,16]$. For example, kidney damage is exacerbated by nephrectomy in streptozotocin-diabetic rats [17] and Zucker fatty rats [18]. Therefore, unilateral nephrectomy is an effective method to accelerate the manifestation of renal alterations. In the present study, we evaluated the effects on renal function of SDT fatty rats subjected to unilateral nephrectomy, as well as the renal morphology of these animals.

Proteinuria, increased blood urea, hypertension, and glomerular sclerosis, which are considered main characteristics of CKD, were observed in the $1 / 2 \mathrm{Nx}$ group at a younger age compared with the sham group.

Compensatory hypertrophy of the remnant kidney after unilateral nephrectomy is well recognized [19]. This phenomenon is accompanied by pathological changes that lead to reduced renal function, although the weight of kidneys has not been used as an indicator of renal dysfunction [19]. In agreement with these findings, the remnant kidney in the $1 / 2 \mathrm{Nx}$ group was significantly heavier than those in the sham group, and histopathological examinations of remnant kidneys in the $1 / 2 \mathrm{Nx}$ group showed degenerative changes such as glomerulosclerosis in the glomerulus and interstitial inflammation in the interstitium. These histopathological findings were not observed in normal Sprague Dawley rats.

Hyperglycemia is a known stimulus for renal hypertrophy and its association with reduction in renal mass affects this hypertrophy $[15,16,20]$. In the present study, levels of plasma glucose were similar among groups. This result suggests that the contribution of hyperglycemia to the exacerbation of renal function in the $1 / 2 \mathrm{Nx}$ group is likely low.

Altered lipid metabolism influences the development and progression of glomerular injury $[21,22]$. Lipid abnormalities 


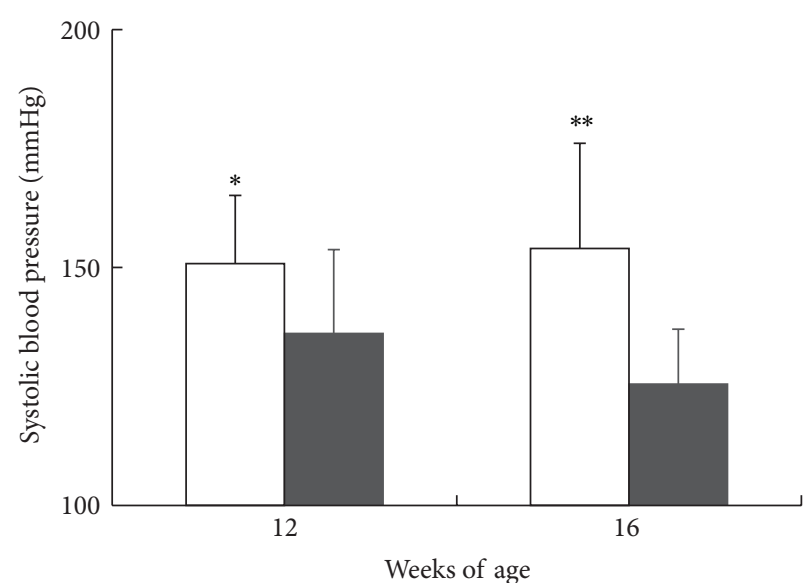

$1 / 2 \mathrm{Nx}$

Sham

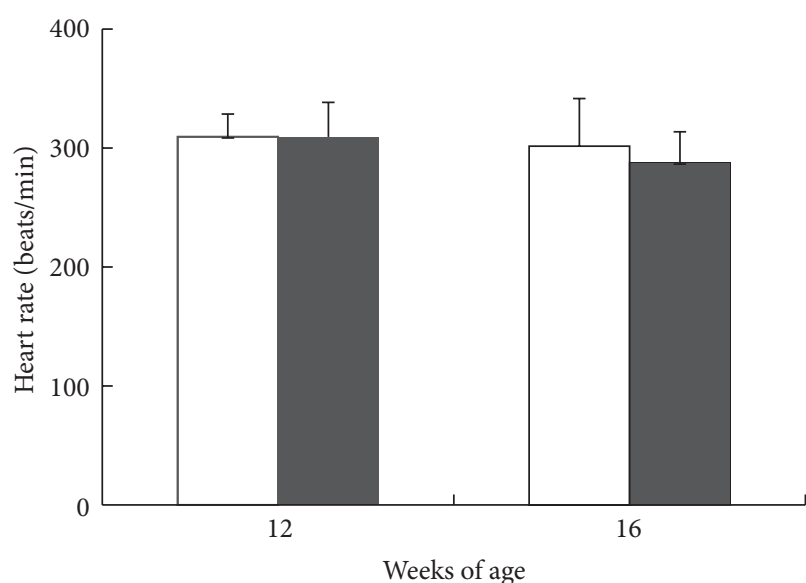

$1 / 2 \mathrm{Nx}$

Sham

(a)

(b)

Figure 2: Systolic blood pressure (a) and heart rate (b) at 12 and 16 weeks of age in the $1 / 2 \mathrm{Nx}$ group and sham group. Data shown as means $\pm \mathrm{SD}(n=8-10) .{ }^{*} P<0.05,{ }^{* *} P<0.01$; significantly different from the sham group.

TABLE 1: Histopathological findings of kidney in sham group and 1/2 Nx group.

\begin{tabular}{|c|c|c|c|c|c|c|c|c|c|c|c|c|c|c|}
\hline \multirow{3}{*}{ Findings } & \multirow[b]{3}{*}{ Animal number } & \multicolumn{13}{|c|}{ Group } \\
\hline & & \multicolumn{8}{|c|}{$1 / 2 \mathrm{Nx}$} & \multicolumn{5}{|c|}{ Sham } \\
\hline & & 1 & 2 & 3 & 4 & 5 & 6 & 7 & 8 & 9 & 10 & 11 & 12 & 13 \\
\hline \multicolumn{15}{|l|}{ Glomerulus } \\
\hline Glomerulosclerosis & & + & + & $2+$ & + & + & $2+$ & + & + & \pm & \pm & \pm & \pm & - \\
\hline \multicolumn{15}{|l|}{ Tubule } \\
\hline Regeneration & & + & $2+$ & $2+$ & $2+$ & $2+$ & $2+$ & $2+$ & $2+$ & + & + & + & + & + \\
\hline Dilatation & & $2+$ & $2+$ & + & $2+$ & + & $2+$ & $2+$ & $2+$ & + & + & \pm & + & + \\
\hline Hyaline cast & & + & + & \pm & \pm & + & \pm & + & + & + & + & \pm & + & \pm \\
\hline Deposit, hyaline droplet & & \pm & - & - & - & - & - & - & - & - & - & - & - & - \\
\hline Armanni-Ebstein change & & + & + & + & + & + & \pm & \pm & - & + & + & + & + & + \\
\hline Mineralization & & - & + & + & + & \pm & + & + & + & + & + & + & - & + \\
\hline \multicolumn{15}{|l|}{ Interstitial } \\
\hline Fibrosis, interstitial & & - & + & - & - & - & + & - & + & - & + & \pm & - & \pm \\
\hline Infiltration, inflammatory cell, interstitial & & + & + & $2+$ & $2+$ & + & $2+$ & $2+$ & $2+$ & \pm & - & + & \pm & + \\
\hline
\end{tabular}

-: negative; \pm : very slight; +: slight; $2+$ : moderate; $3+$ : severe.

in the $1 / 2$ Nx group, which accompany a reduction in renal mass, may contribute to progressive glomerular damage.

Hypertension is a hemodynamic characteristic of CKD, which could accelerate the progression of renal dysfunction by worsening glomerular injury and proteinuria [23]. It is possible that the increased blood pressure observed in the $1 / 2 \mathrm{Nx}$ group in this study contributed to the accelerated glomerular injury and consequently led to marked proteinuria. Proteinuria has been considered a strong predictor of kidney disease outcome [24]. In addition, urinary biomarkers such as kidney injury molecule-1 (KIM-1) and N-acetyl- $\beta$-Dglucosaminidase (NAG) have been reported $[25,26]$. In the further study, measuring these biomarkers may be useful to assess the severity of diabetic kidney damage.
In conclusion, induction of $1 / 2 \mathrm{Nx}$ in SDT fatty rats led to functional and morphological damage of the remnant kidney and to hypertension, which are considered main characteristics of chronic kidney disease, at a younger age. The early onset of diabetic nephropathy in SDT fatty rats is an advantage for CKD research. The SDT fatty rat has promise in the further elucidation of the pathogenesis of human diabetic nephropathy and in new drug discovery.

\section{Conflict of Interests}

The authors declare that there is no conflict of interests regarding the publication of this paper. 


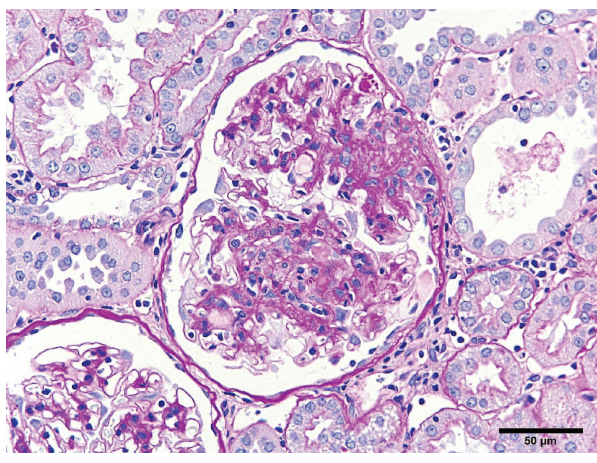

(a)

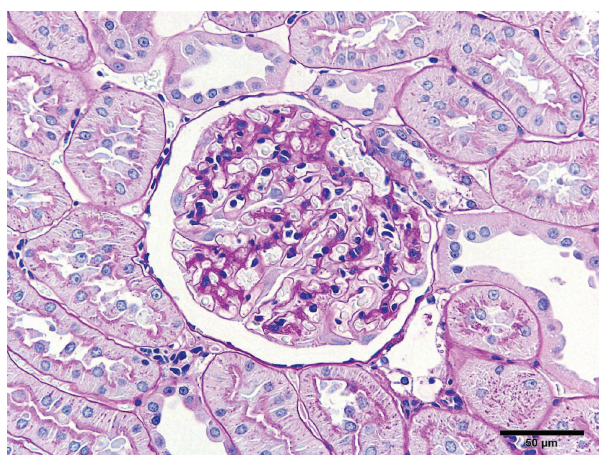

(c)

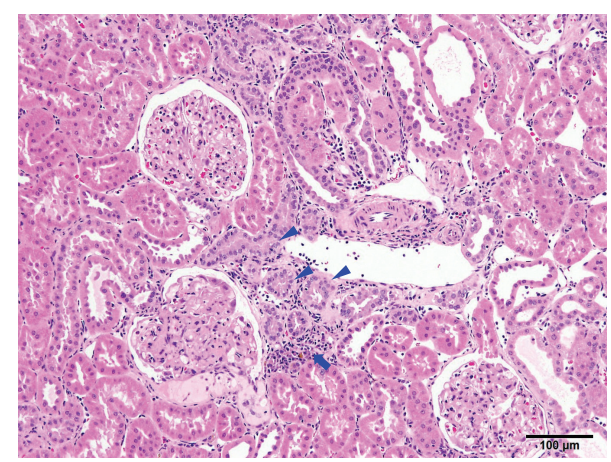

(b)

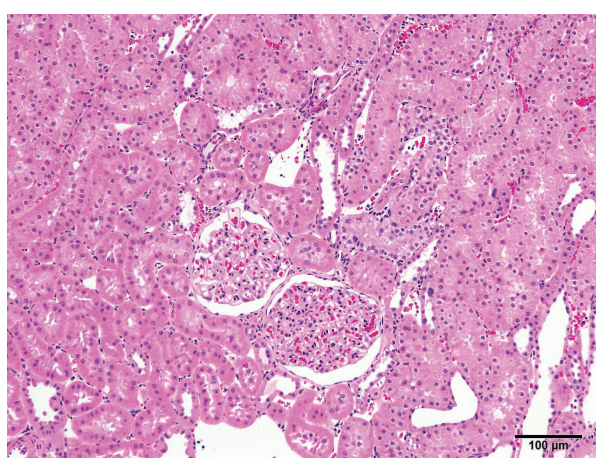

(d)

Figure 3: Photomicrograph of kidney tissues from the 1/2 Nx group (a) and (b) and sham group (c) and (d). Marked glomerulosclerosis (a) tubular regeneration (arrowhead in (b)) and inflammatory cell infiltration (arrow in (b)) observed in the 1/2 Nx group. Periodic acid Schiff ((a) and (c)), bar $=50 \mu \mathrm{m}$. Hematoxylin and eosin ((b) and (d)). Bar $=100 \mu \mathrm{m}$.

\section{Authors' Contribution}

Y. Katsuda, K. Kakimoto, and T. Ohta helped in the design of the study. Y. Katsuda, Y. Kemmochi, M. Maki, R. Sano, Y. Toriniwa, Y. Ishii, and K. Miyajima helped in the data collection and conduct of the study. Y. Katsuda, Y. Kemmochi, M. Maki, and T. Ohta helped in the analysis of study. Y. Katsuda and $\mathrm{T}$. Ohta helped in writing the paper.

\section{References}

[1] D. L. Hoyert, M. P. Heron, S. L. Murphy, and H. C. Kung, "Deaths: final data for 2003," National Vital Statistics Reports, vol. 54, no. 13, pp. 1-120, 2006.

[2] E. S. Schaeffner, T. Kurth, G. C. Curhan et al., "Cholesterol and the risk of renal dysfunction in apparently healthy men," Journal of the American Society of Nephrology, vol. 14, no. 8, pp. 20842091, 2003.

[3] R. Agarwal, "Blood pressure components and the risk for endstage renal disease and death in chronic kidney disease," Clinical Journal of the American Society of Nephrology, vol. 4, no. 4, pp. 830-837, 2009.

[4] T. M. Islam, C. S. Fox, D. Mann, and P. Muntner, "Age-related associations of hypertension and diabetes mellitus with chronic kidney disease," BMC Nephrology, vol. 10, no. 1, article 17, 2009.

[5] E. Ritz and A. Stefanski, "Diabetic nephropathy in type II diabetes," The American Journal of Kidney Diseases, vol. 27, no. 2, pp. 167-194, 1996.
[6] J. E. Hall, J. J. Kuo, A. A. da Silva, R. B. de Paula, J. Liu, and L. Tallam, "Obesity-associated hypertension and kidney disease," Current Opinion in Nephrology and Hypertension, vol. 12, no. 2, pp. 195-200, 2003.

[7] J. Petersen, J. Ross, and R. Rabkin, "Effect of insulin therapy on established diabetic nephropathy in rats," Diabetes, vol. 37, no. 10, pp. 1346-1350, 1988.

[8] K. Matsui, T. Ohta, H. Morinaga et al., "Effects of preventing hyperphagia on glycolipid metabolic abnormalities in Spontaneously Diabetic Torii fatty rats," Animal Science Journal, vol. 79, no. 5, pp. 605-613, 2008.

[9] K. Matsui, T. Ohta, T. Oda et al., "Diabetes-associated complications in Spontaneously Diabetic Torii fatty rats," Experimental Animals, vol. 57, no. 2, pp. 111-121, 2008.

[10] H. Morinaga, T. Ohta, K. Matsui et al., "Effect of food restriction on adipose tissue in spontaneously diabetic Torii fatty rats," Experimental Diabetes Research, vol. 2009, Article ID 715057, 9 pages, 2009.

[11] Y. Ishii, T. Ohta, T. Sasase et al., "Pathophysiological analysis of female spontaneously diabetic torii fatty rats," Experimental Animals, vol. 59, no. 1, pp. 73-84, 2010.

[12] Y. Katsuda, T. Ohta, K. Miyajima et al., "Diabetic complications in obese type 2 diabetic rat models," Experimental Animals, vol. 63, no. 2, pp. 121-132, 2014.

[13] Y. Ishii, M. Maki, H. Yamamoto, T. Sasase, M. Kakutani, and T. Ohta, "Evaluation of blood pressure in spontaneously diabetic torii-Leprfa rats," Experimental Animals, vol. 59, no. 4, pp. 525529, 2010. 
[14] Y. Ishii, M. Maki, H. Yamamoto, T. Sasase, M. Kakutani, and T. Ohta, "Blood pressure characteristics of female spontaneously diabetic Torii-Leprfa rats," Journal of Veterinary Medical Science, vol. 73, no. 4, pp. 501-505, 2011.

[15] M. W. Steffes, D. M. Brown, and S. M. Mauer, "Diabetic glomerulopathy following unilateral nephrectomy in the rat," Diabetes, vol. 27, no. 1, pp. 35-41, 1978.

[16] M. P. O’Donnell, B. L. Kasiske, F. X. Daniels, and W. F. Keane, "Effects of nephron loss on glomerular hemodynamics and morphology in diabetic rats," Diabetes, vol. 35, no. 9, pp. 1011$1015,1986$.

[17] G. S. Lopes, C. C. S. Lemos, C. A. Mandarim-de-Lacerda, and R. Bregman, "Effect of unilateral nephrectomy on renal function of diabetic rats," Histology and Histopathology, vol. 19, no. 4, pp. 1085-1088, 2004.

[18] B. L. Kasiske, M. P. O’Donnell, M. P. Clearly, W. F. Keane, and F. Phillips, "Effects of reduced renal mass on tissue lipids and renal injury in hyperlipidemic rats," Kidney International, vol. 35, no. 1, pp. 40-47, 1989.

[19] V. Oršić, M. Mihalj, M. Moguš et al., "Impaired kidney function in rats six months after unilateral nephrectomy: an old story, a new perspective," Medicinski Glasnik, vol. 8, no. 2, pp. 185-191, 2011.

[20] T. H. Hostetter, J. L. Troy, and B. M. Brenner, "Glomerular hemodynamics in experimental diabetes mellitus," Kidney International, vol. 19, no. 3, pp. 410-415, 1981.

[21] L. Peric Golia and M. Peric Golia, "Aortic and renal lesions in hypercholesterolemic adult, male, virgin Sprague-Dawley rats," Atherosclerosis, vol. 46, no. 1, pp. 57-65, 1983.

[22] B. L. Kasiske, M. P. O’Donnell, W. J. Garvis, and W. F. Keane, "Pharmacologic treatment of hyperlipidemia reduces glomerular injury in rat 5/6 nephrectomy model of chronic renal failure," Circulation Research, vol. 62, no. 2, pp. 367-374, 1988.

[23] R. F. Gagnon and M. Ansari, "Development and progression of uremic changes in the mouse with surgically induced renal failure," Nephron, vol. 54, no. 1, pp. 70-76, 1990.

[24] J. C. Peterson, S. Adler, J. M. Burkart et al., "Blood pressure control, proteinuria, and the progression of renal disease: the modification of diet in renal disease study," Annals of Internal Medicine, vol. 123, no. 10, pp. 754-762, 1995.

[25] C. C. Luka, K. Chowa, J. S. Kwok et al., "Urinary biomarkers for the prediction of reversibility in acute-on-chronic renal failure," Disease Markers, vol. 34, no. 3, pp. 179-185, 2013.

[26] F. L. Nauta, W. E. Boertien, S. J. L. Bakker et al., "Glomerular and tubular damage markers are elevated in patients with diabetes," Diabetes Care, vol. 34, no. 4, pp. 975-981, 2011. 


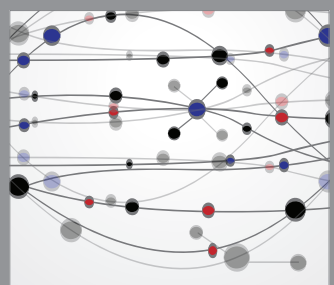

The Scientific World Journal
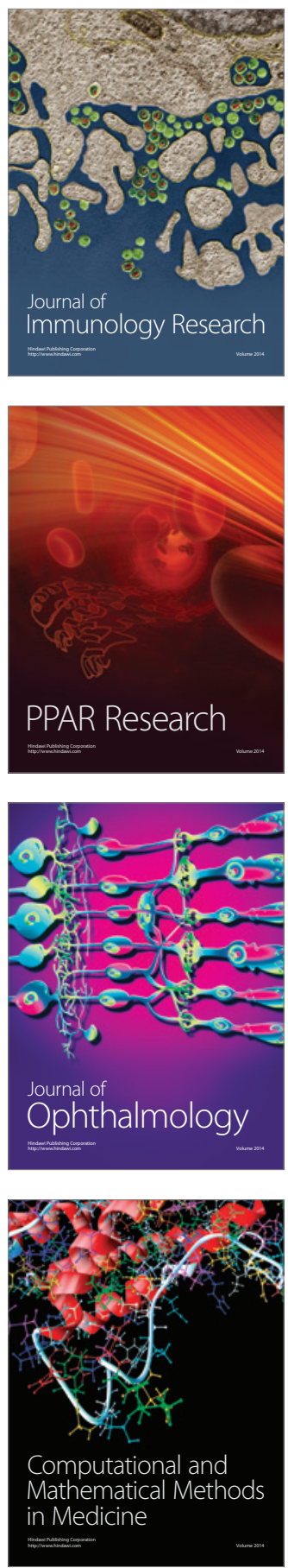

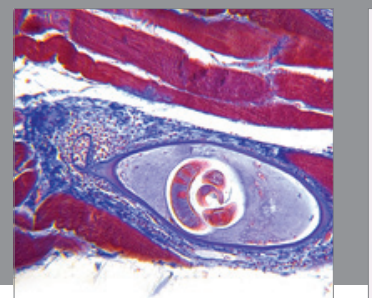

Gastroenterology

Research and Practice
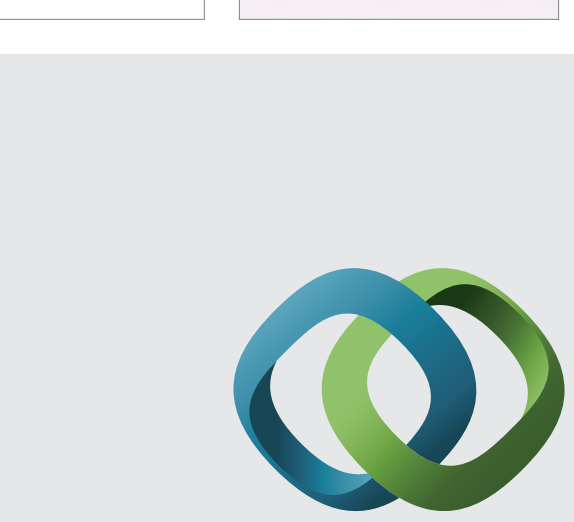

\section{Hindawi}

Submit your manuscripts at

http://www.hindawi.com
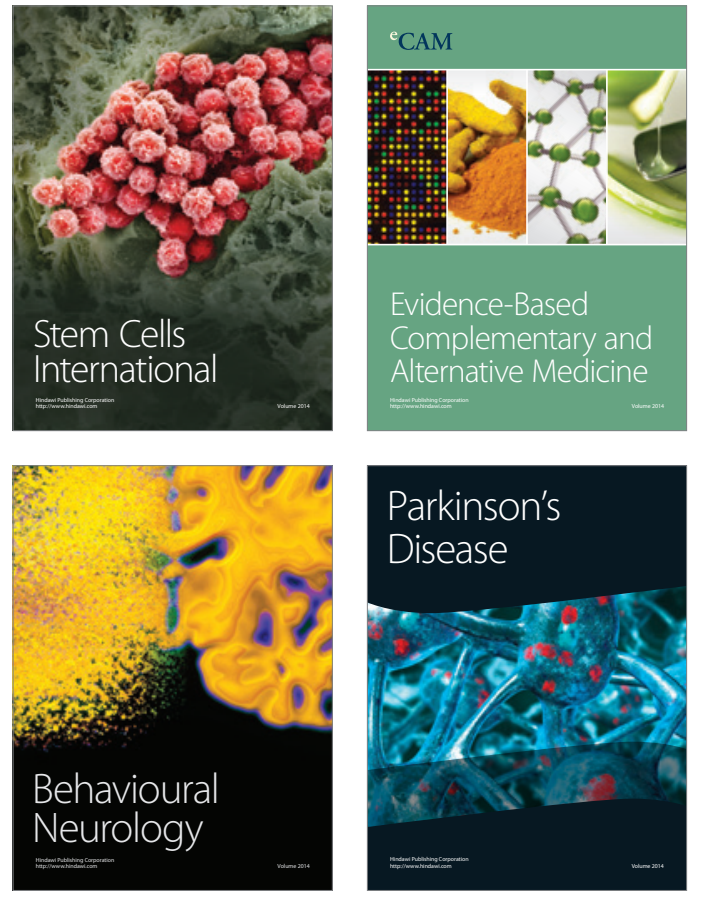
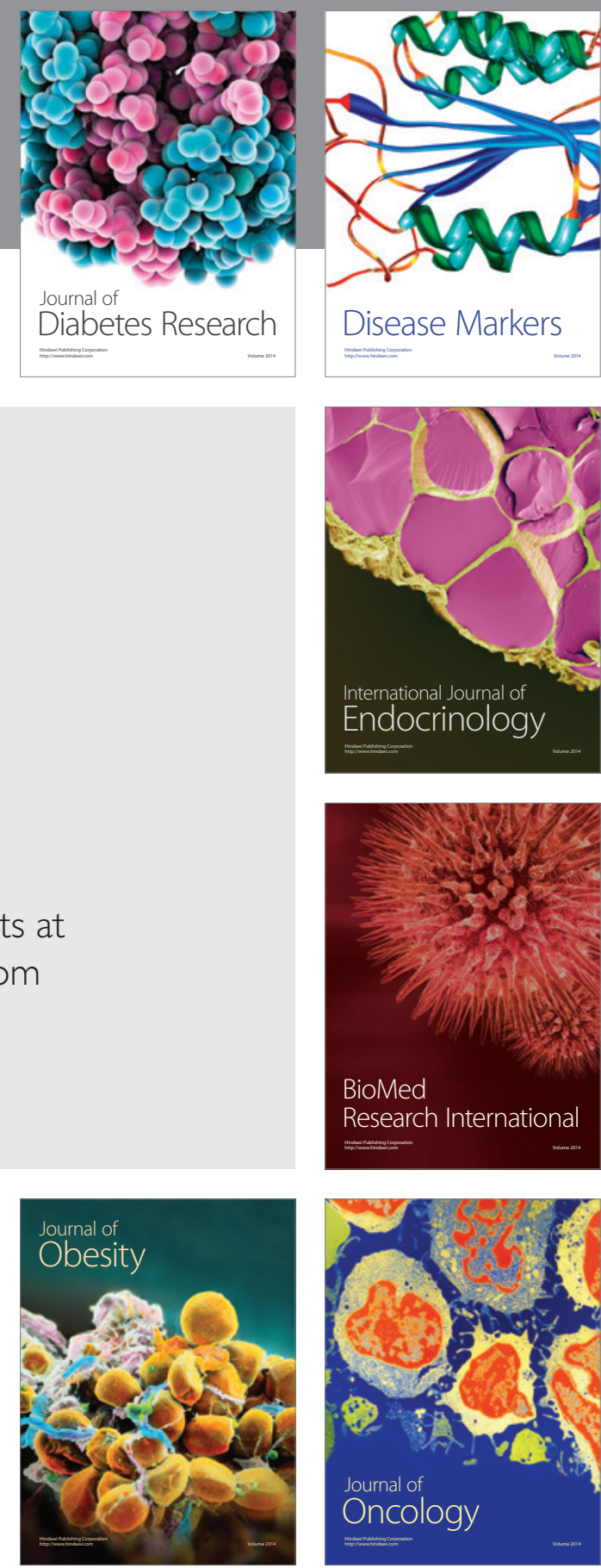

Disease Markers
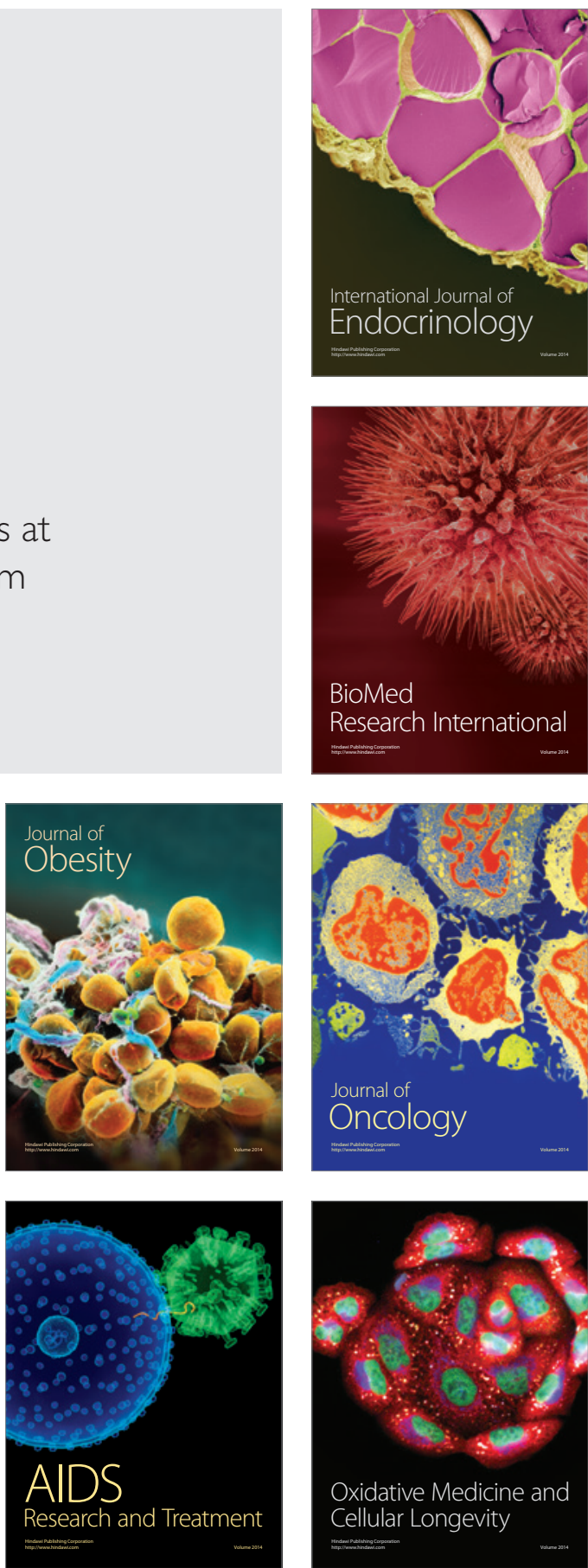